\title{
Uterine Corpus Carcinoma and Carcinosarcoma pN1 TNM Finding v8
}

National Cancer Institute

\section{Source}

National Cancer Institute. Uterine Corpus Carcinoma and Carcinosarcoma pN1 TNM

Finding v8. NCI Thesaurus. Code C139780.

Uterine corpus carcinoma or carcinosarcoma with regional lymph node metastasis to pelvic lymph nodes. (from AJCC 8th Ed.) 